\title{
Factores asociados a depresión posnatal en puérperas de Antioquia, según escala de Edimburgo
}

\section{Factors associated with postnatal depression in puerperal women in Antioquia, according to the Edinburgh scale}

\author{
Gustavo Edgardo Jiménez-Hernández ${ }^{1}{ }^{*}$, Yesenia Margarita Peña Jaramillo ${ }^{1} \mathcal{D}$, Mariana Ortega Pérez ${ }^{1}$ \\ D, Julieth Gil Salazar ${ }^{1}$, Katherine Arias Vélez ${ }^{1}$ \\ ${ }^{1}$ Universidad Católica de Oriente, Rionegro, Colombia \\ * Dirigir correspondencia a: gjimenezhdz@yahoo.es
}

Proceso editorial

Recibido: 060220

Aceptado: 280720

Publicado: 140820

DOI 10.17081/innosa.84

(C) Copyright 2020

Jiménez-Hernández

${ }^{1}$ et al.

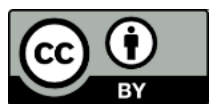

\section{RESUMEN}

Introducción: La depresión postparto (DPP) es una complicación que se presenta con frecuencia en mujeres durante este periodo, y representa un riesgo para la salud en la diada madre - hijo. Objetivo: Determinar la prevalencia y los factores asociados de la depresión posparto en Colombia. Materiales y métodos: Se trata de un estudio descriptivo de corte transversal, se registraron 203 mujeres residentes en el departamento de Antioquia - Colombia, quienes tuvieron el parto entre 2017 - 2018 y se les aplicó la escala de Edimburgo hasta los 6 meses luego del parto. Resultados: La mediana de edad de las mujeres estudiadas fue de 25 años, rango intercuartílico (RIQ 21-30 años), El 45,1 \% viven en zona rural, el 64,5\% tuvo un grado de escolaridad como bachiller, el 20,27\% presentó puntajes $\geq 10$ como indicador probable de DPP, el 23,2 \% presentó historia personal de depresión, y el 26,7\% confirmó antecedentes familiares de depresión. Conclusiones: Se logró describir y asociar algunas variables epidemiológicas y las incluidas en la escala de Edimburgo para depresión posparto. Se evidenció que los trastornos psicológicos, los antecedentes personales y familiares de depresión, estuvieron asociados en el desarrollo de depresión posparto.

Palabras clave: Depresión postparto; Variables Epidemiológicas; Salud Mental; Escalas de valoración Psiquiátrica

\section{ABSTRACT}

Introduction: Postpartum depression (PPD) is a complication that often occurs in women during this period and represents a health risk in the dyad mother - child. Objective: To determine the prevalence and associated factors of postpartum depression in Colombia. Materials and methods: This is a descriptive cross-sectional study, 203 women residents in the department of Antioquia - Colombia were registered, who had the birth between 2017 - 2018 and the Edinburgh scale was applied up to 6 months after delivery. Results: The median age of the women studied was 25 years, interquartile range (RIQ 21-30 years), 45,9\% live in rural areas, 64.5 $\%$ had a bachelor's degree, $20.27 \%$ had scores $\geq 10$ as a probable indicator of PPD, $23.2 \%$ had a personal history of depression, and $26.7 \%$ confirmed a family history of depression. Conclusions: Some epidemiological variables and those included in the Edinburgh scale for postpartum depression are described and associated. Psychological disorders, personal and family history of depression, those associated with the development postpartum depression are evident.

Keywords: Postpartum depression; Epidemiological variables; Mental Health; Psychiatric assessment.

Cómo citar: Jiménez-Hernández G, Peña Y, Ortega M, Gil J, Arias K. Factores asociados a depresión posnatal en puérperas de Antioquia, según escala de Edimburgo. Ciencia e Innovación en Salud. 2020. e84: 285-296. DOI 10.17081/innosa.84 


\section{INTRODUCCIÓN}

La depresión posparto (DPP) es un importante problema de salud materna que ocurre durante el primer año después del parto, sus síntomas incluyen cambios en los patrones del sueño, alimentación, fatiga, tristeza, llanto, ansiedad y sentimientos de culpa relacionados con la capacidad de cuidar a su hijo (1). De acuerdo con el manual estadístico y de diagnóstico estandarizado (DSM-5) (2)), la depresión posparto es un tipo de episodio depresivo, que ocurre en este periodo de la vida, inicia durante el embarazo y puede prolongarse hasta por más de seis meses después del nacimiento del bebé, y si no es tratada puede persistir la sintomatología hasta por un año en un $25 \%$ de los casos(므). La DPP es un problema que afecta alrededor del $17 \%$ de las madres en todo el mundo y en países de ingresos bajos y medios hasta el $19 \%$ (1)(4) Otro estudio publicado en el British Journal of Psychiatry en 2017, reveló que la prevalencia de la DPP fue entre el $13-40 \%(\underline{5})$

Los resultados publicados en la Primera Encuesta Nacional de Salud Mental en Colombia durante el año 2015, mostró que entre un 8 y un 25\% de las mujeres presentan sintomatología asociadas a síndrome depresivo durante el periodo del postparto, y la depresión puede alcanzar hasta un $66 \%$ del total de las mujeres durante el puerperio. (ㄱ). Por lo tanto, es importante estimar de manera confiable la depresión posparto en estos contextos, con el propósito de generar nuevas políticas en salud mental.

La DPP no solo trae consecuencias negativas para la salud física de la mujer, también puede afectar la capacidad y calidad del cuidado del niño (4) Esto a su vez puede desencadenar trastornos cognitivos, de conducta, de interacción social y un desarrollo físico deficiente durante

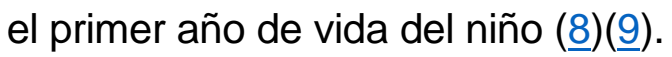

Investigaciones previas han revelado que factores como, la ansiedad durante el embarazo, situaciones recientes de estrés, falta de apoyo social, situaciones de abuso, bajo nivel educativo y bajo nivel socioeconómico, están asociadas con la presencia de DPP (1)(10)(11). Sin embargo, también se han desarrollado herramientas para prevenir, diagnosticar y tratar este problema (12) Para ello, se han diseñado varias escalas útiles en el tamizaje, entre las que se destaca por su utilidad práctica la Escala de Depresión Posparto de Edimburgo (EDPPE), creada a finales de la década de los 80 en Inglaterra por Cox y col. (13), como una herramienta diagnostica eficaz de fácil aplicación permitiendo la identificación oportuna de las variables y sintomatología relacionadas a trastorno depresivo postparto.

La Escala de Depresión Postnatal de Edimburgo (EPDS) se considera el estándar de oro en la detección de la depresión posparto. La versión en español ha sido ampliamente utilizada. En Colombia se realizó un estudio donde se evaluó su consistencia interna y su estructura factorial en la que se indicó un modelo con tres factores consistentes, la ansiedad, depresión y anhedonia, los resultados mostraron que la escala de Edimburgo para depresión posparto posee una alta consistencia interna y una estructura tridimensional para el concepto de depresión en 
mujeres embarazadas de Colombia $(\underline{14})(\underline{15})$. El objetivo de este estudio fue determinar la prevalencia y los factores asociados de la depresión posparto en el departamento de Antioquia.

\section{MÉTODOS}

Se trata de un estudio descriptivo de corte transversal. Se desarrolló a partir de una base hospitalaria con 203 partos atendidos entre los años 2017 y 2018 en dos hospitales del departamento de Antioquia. El diseño muestral fue no probabilístico por conveniencia. Previa autorización para el uso de datos se procedió a contactar cada una de las mujeres atendidas y se les explicó el propósito del estudio, que implicó mínimo riesgo y el mantenimiento del anonimato de acuerdo con las disposiciones colombianas para investigaciones en salud. La participación fue de manera voluntaria y se les solicitó la firma del consentimiento informado.

De las 203 puérperas, 113 (69,3\%) fueron incluidas en el estudio, a 21 se les aplicó el instrumento vía telefónica por difícil acceso al domicilio, y a 92 se les recolectó la información de manera presencial mediante visita domiciliaria. El 30,7\% $(n=90)$ restante de la muestra se excluyó por datos erróneos de contacto $(n=28)$, y porque no aceparon participar en el estudio $(n=62)$.

Luego de la revisión de la historia clínica, se aplicó la Escala de Edimburgo dentro de los primeros seis meses del parto.

La Escala de Depresión Postnatal de Edimburgo es un instrumento autoadministrado, consta de diez ítems de respuesta politómica (cuatro opciones de respuesta) que exploran síntomas cognoscitivos de un episodio depresivo mayor durante los últimos quince días. Cada pregunta se califica de cero a tres puntos de acuerdo con la severidad creciente de los síntomas. Requiere únicamente cinco a diez minutos para diligenciarse. Los puntos de corte iguales o superiores a 10 se consideraron sospecha de DPP. Se incluyeron datos sociodemográficos en el instrumento para caracterizar a la población estudiada.

La información recolectada se iba almacenando y codificando en un libro de EXCEL (Microsoft office Excel 2016) por dos investigadores con el fin de evitar errores de digitación. En primera instancia se realizó estadística descriptiva. Para variables continuas se calcularon la media, desviación estándar, RIQ (rango intercuartílico) y t de student. Para las variables cualitativas que componen la escala de Edimburgo, se calcularon las respectivas proporciones, además se aplicó el , test exacto de Fisher y Chi cuadrado de Pearson para el análisis bivariado, comparando las diferencias entre lo observado y lo esperado, la significancia estadística fue considerada con un valor de $p<0,05$. Para el análisis estadístico se utilizó el software IBM Statistical Package for the Social Sciences SPSS Statistics 22 (IBM Corporation, Chicago, Delaware.,USA) (Agosto 2017). 


\section{RESULTADOS}

La media de edad de las mujeres estudiadas fue de 25 años, con una DE +/- 6,2 un rango intercuartílico de (RIQ 21-30 años), el 55,3\% procedían del área urbana, el nivel educativo de bachiller fue en mayor proporción el alcanzado con $65,1 \%$, el 71,4 \% no estaba laborando al momento de la encuesta. El nivel socioeconómico 1 y 2 fueron los que en mayor magnitud pertenecieron las mujeres estudiadas, con un $14,2 \%$ y $71,4 \%$ respectivamente. El $46,9 \%$ comunicó su estado civil de casada, además el 61,1\% manifestó tener problemas de convivencia durante la gestación y al momento de la planificación del embarazo. Los demás detalles de estadística descriptiva para las diferentes variables se encuentran en la Tabla I.

Para el análisis bivariado, se dividió la muestra en dos grupos, el primero con quienes obtuvieron un puntaje de corte menor a 10 en la escala (sin riesgo de depresión posparto) $79,6 \% \mathrm{n}=(90)$, y el segundo grupo quienes obtuvieron puntajes iguales o superiores a 10 puntos (riesgo de desarrollar depresión posparto) 20,3\% $n=(\underline{23})$.

Al aplicar los estadísticos de prueba en el análisis bivariado, los resultados mostraron que hubo diferencia entre los grupos al asociarlo con los antecedentes de trastornos psicológicos, con un $p$ - valor $=0,01$, y el historial de depresión en parientes de primer grado con un $p$-valor $=0,04$. Las demás variables no fueron estadísticamente significativas. Los resultados del análisis bivariado aparecen con detalle en la Tabla 1. Observación:

Tabla 1. Descripción y comparación de características sociodemográficas entre grupos

\begin{tabular}{lccc} 
& $\begin{array}{c}\text { Puntaje de } \\
\text { Edimburgo } \leq 10\end{array}$ & $\begin{array}{c}\text { Puntaje de } \\
\text { Edimburgo } \geq 10\end{array}$ & p - valor \\
\hline $\begin{array}{l}\text { Edad Promedio + DS } \\
\text { Procedencia }\end{array}$ & $25,2+/-6,2(90)$ & $26,5+/-5,8(23)$ & $0,658^{*}$ \\
Urbana & $40,7 \% \mathrm{n}=(46)$ & $14,1 \% \mathrm{n}=(16)$ & $0,159^{* * *}$ \\
Rural & $38,9 \% \mathrm{n}=(44)$ & $6,2 \% \mathrm{n}=(7)$ & \\
Nivel_Educativo & $0,88 \% \mathrm{n}=(1)$ & $\mathrm{n}=(0)$ & \\
Sin estudios & $24,8 \% \mathrm{n}=(28)$ & $4,42 \% \mathrm{n}=(5)$ & \\
Básica primaria & $49,5 \% \mathrm{n}=(56)$ & $15,0 \% \mathrm{n}=(17)$ & \\
Secundaria & $4,42 \% \mathrm{n}=(5)$ & $0,88 \% \mathrm{n}=(1)$ & \\
Universidad & & & \\
& & & \\
Estado Laboral & $21,2 \% \mathrm{n}=(24)$ & $7,07 \% \mathrm{n}=(8)$ & \\
Trabaja & $58,4 \% \mathrm{n}=(66)$ & $13,2 \% \mathrm{n}=(15)$ & \\
No trabaja & & & $0,287^{* * *}$ \\
Estado Civil & $39,8 \% \mathrm{n}=(45)$ & $7,07 \% \mathrm{n}=(8)$ & \\
Casada & &
\end{tabular}


Con pareja estable

Soltera

Nivel socieconomico

NSE 1

NSE 2

NSE 3

NSE 4

$$
\begin{aligned}
& 27,4 \% n=(31) \\
& 12,4 \% n=(14)
\end{aligned}
$$

Perdida del trabajo durante el embarazo

SI

$\mathrm{NO}$

$$
n=(0)
$$

$79,6 \% \mathrm{n}=(90)$

$$
22,1 \% n=(25)
$$

$15,0 \% n=(17)$

$15,0 \% \mathrm{n}=(17)$

Nada

Problemas de convivencia durante la gestación y la planificación del embarazo

Si

No

Problemas

Psicológicos

$\mathrm{Si}$

No
$10,6 \% n=(12)$

$2,6 \% n=(3)$

$$
5,3 \% n=(6)
$$

$12,4 \% \mathrm{n}=(14)$

$$
2,6 \% n=(3)
$$

$$
\mathrm{n}=(0)
$$

$0,204^{\star * *}$

$0,88 \% n=(1)$

$19,5 \% n=(22)$

$0,244^{* * *}$

$$
8,0 \% n=(9)
$$$$
2,6 \% n=(3)
$$

$7,07 \% n=(8)$

$2,6 \% n=(3)$

$0,392^{* * *}$
$12,4 \% \mathrm{n}=(14)$

$8,0 \% \mathrm{n}=(9)$ $0,983^{\star * \star}$

$0,01^{\star * *}$

$8,0 \% n=(9)$

$12,4 \% \mathrm{n}=(14)$
$\mathrm{Si}$

No

$$
7,07 \% \mathrm{n}=(8)
$$$$
72,6 \% n=(82)
$$

Historial familiar de depresión en parientes de primer grado

$9,7 \% \mathrm{n}=(11)$ $10,6 \% n=(12)$
$\mathrm{Si}$

No

Enfermedades Crónicas

$\mathrm{Si}$

No
$17,7 \% \mathrm{n}=(20)$

$62,0 \% \mathrm{n}=(70)$

$19,5 \% \mathrm{n}=(22)$

$60,2 \% n=(68)$

Número de abortos anteriores

$\mathrm{Si}$

No

Complicaciones durante la gestación y el parto

$\mathrm{Si}$

No
$16,8 \% \mathrm{n}=(19)$

$62,8 \% n=(71)$
$8,8 \% \mathrm{n}=(10)$

$11,5 \% n=(13)$

$0,473^{* * *}$

$3,5 \% \mathrm{n}=(4)$

$16,8 \% n=(19)$

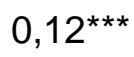

$10,6 \% \mathrm{n}=(12)$

$9,7 \% n=(11)$

$0,17^{* * *}$

$7,07 \% \mathrm{n}=(8)$

$13,3 \% n=(15)$

*Test de Student ${ }^{* * *}$ Test Chi cuadrado. Fuente: Elaboración propia*** 


\section{DISCUSIÓN}

La investigación arrojó que el 20,27 \% de las mujeres participantes tuvieron puntos de corte mayores o iguales a 10, lo que corresponde a haber presentado depresión posparto. El hallazgo es similar en los datos obtenidos por otros autores en estudios realizados en Venezuela (17), con un $22 \%$. Esto podría deberse a la similitud metodológica y al uso de la misma escala como herramienta, con un punto de corte igual, que es un EPDS $\geq 10$. Mostrando así que esta patología se encuentra presente en un gran número de mujeres y que en la mayoría de los casos pudiese tratarse de un evento subdiagnosticado y subtratado.

La edad media de las mujeres estudiadas fue de 25 años, (rango intercuartílico entre 21-30 años), la variable edad no arrojó significancia estadística al asociarla con los puntajes de corte para depresión, $\mathrm{p}$-valor $=0,658$. Sin embargo, los estudios han mostrado discrepancia en cuanto a la edad como factor de riesgo para desarrollar este evento, por ejemplo, se ha reportado que la DPP fue más común entre las mujeres de edades comprendidas entre 18 y 23 años, comparadas con los grupos de mujeres mayores de treinta años o más (1).Los autores reportaron que pudo deberse a que las mujeres más jóvenes están más expuestas a la angustia emocional, por la poca experiencia del parto y se adiciona una carga del cuidado para sus hijos, así como para toda su familia durante este período. Otros estudios han mostrado que, las mujeres mayores de 30 años tuvieron mayor riesgo de desarrollar depresión posparto respecto a las mujeres más jóvenes(20), estos resultados son similares a estudios realizados en Nepal (21), Finlandia (22), China (23) y EE. UU.(24). Los autores explicaron que el posible decremento de la depresión posparto en la edad temprana pudo deberse al aumento de la cobertura educativa en estos países. Asimismo en España un estudio mostr que las edades de las mujeres que obtuvieron puntuaciones > 13 en la EPDS, fue de 30 años IC95\% $(28,8-31,5)$ con un p valor de $0,05(\underline{25})$, otra investigación realizada en Venezuela mostró que la edad promedio fue de 24 años con rangos entre 19 - 33 años (17).

El 54,8 \% de la muestra estudiada procedieron del área urbana, de ellas solo un $14,1 \%$ obtuvo un puntaje > 10 en la escala, mientras que este punto de corte solo representó un 6,2\% para quienes viven en el área rural. En un estudio realizado en Colombia arrojó que la depresión posparto representó mayor riesgo de presentarlo en mujeres que viven en zonas urbanas (26). Esto se deba a que probablemente en el área urbana las mujeres estén más vulnerables a exigencias de tipo social, que conlleven a experimentar deterioro de su imagen corporal y requiera volver a su estado anterior de manera rápida, y desde lo laboral, por la necesidad de una reincorporación a su vida productiva para generar ingresos asumiendo responsabilidades de nuevos gastos por su hijo, experimentando estrés o sentimientos de dependencia si es el caso.

Al comparar los grupos de riesgo de desarrollar DPP y el nivel socioeconómico no se apreció significancia estadística $p$-valor $=0,392$. Sin embargo, en otros estudios los resultados han mostrado que hubo mayor riesgo entre las participantes que tenían bajos ingresos en 
comparación con las mujeres que tenían un ingreso mayor. Ejemplo de ello fueron estudios

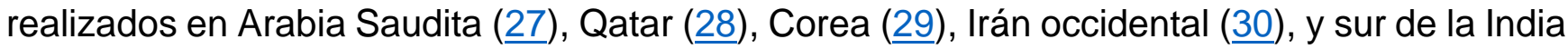
(31).

El nivel educativo fue otra variable que no mostró significancia estadística con la DPP $p$ - valor $=0,742$. El $15 \%$ de la muestra estudiada presento riesgo de desarrollar DPP, con nivel educativo de secundaria, y el $4,42 \%$ de básica primaria. El estudio de Urdaneta J, realizado en Venezuela reportó que las mujeres con un nivel educativo primario o inferior presentaron mayor riesgo de desarrollar depresión posparto, mientras que un nivel educativo superior se comportó como efecto protector, p-valor $=0,05$ (17). En Chile, Rojas y cols. (르) reportaron una correlación de mayor gravedad en la sintomatología según el puntaje de la escala con menor grado educativo; Igualmente Alvarado y cols. comunicaron frecuencias significativas mayores de DPP en mujeres con hasta 6 años de educación que en aquellas con más de 6 años , p-valor $=0,0009)(\underline{33})$.

En cuanto a la situación laboral no se encontró diferencias entre los grupos, aunque el $71.7 \%$ de las mujeres encuestadas no trabajaba, se dedican a tareas domésticas, condición que las hacen propensas para depresión (34); Warner et al y Cooper et al, citados por Sierra Manzano JM, Carro T y Ladrón E, evidenciaron en sus estudios que el desempleo se relaciona de forma consistente con el riesgo de depresión posparto (25). De otra parte F Machado Ramírez et al. (35), encontraron que en las mujeres que trabajan fuera del hogar, tiene incidencia el nivel socioeconómico así: las de los niveles 4 y 5 presentan mayor riesgo de depresión posparto que las mujeres de los niveles 1, 2 y 3 , también encontraron que no disfrutar de la baja maternal constituye un factor de riesgo para la depresión posparto, un $40 \%$ de quienes declararon no disfrutar de ella obtuvieron una puntuación igual o superior a 13 en el EPDS, mientras que de 117 mujeres que si disfrutaron de baja maternal la sospecha de depresión fue del 12.8\% ( $p=0,01)$ ( $\underline{6}$ ). Estos estudios mostraron que las amas de casa de tiempo completo son más propensas a la depresión posparto; señalando que está asociado al riesgo de depresión posparto tanto el hecho de no trabajar fuera de la casa $(p<0,05)$ como la pérdida del empleo durante la gestación $(p<0.01)(\underline{16})$.

En relación con los problemas de convivencia durante la gestación, relacionada con la planificación de la última gestación, se encontró que estas variables no mostraron significancia estadística para la presencia de depresión posparto $(p=0.983)$, solo el $12,4 \%$ de las mujeres encuestadas que habían planificado su embarazo presentaron una puntuación mayor de 10, en total el $61,1 \%$ reportó que su última gestación fue planificada, similar a los resultados obtenidos en el estudio realizado por Rosa González Navarrete y Ligia García López en Yucatán, en el cual el $58 \%$ de los casos tenían su última gestación planeada ( $\underline{36})$, lo que muestra que las decisiones de tener hijos o no está dividida entre las parejas actuales.

En este estudio la variable relacionada con el apoyo de la pareja no resultó significativa, el $9,72 \%$ de las mujeres encuestadas que gozaron con poca o ninguna ayuda de su pareja tuvieron una puntuación de $>10$; en contraste con otros estudios se considera que esta 
variable abarca no solo la ayuda, sino la relación con la pareja y con otros miembros de la familia. Un estudio de cohorte realizado en Australia arrojó que apoyo social antes y después del parto es un posible factor protector tanto para la depresión perinatal, como para el desarrollo infantil adverso, que es una consecuencia conocida de la depresión perinatal. Se encontró además que las relaciones entre depresión y ansiedad y los resultados del niño eran en gran medida independiente del apoyo social. Sin embargo, estos resultados apoyan la importancia de realizar investigaciones adicionales en ajustar los enfoques actuales de tratamiento para la depresión perinatal para que sean más orientados al apoyo social desde el final del embarazo y durante los primeros seis meses posparto ( $\underline{37})$

En el análisis bivariado los trastornos psicológicos y psiquiátricos mostraron significancia frente a la depresión posparto, $p$ - valor $=0.01$. Estos resultados coinciden con otros estudios que evidenciaron los antecedentes psiquiátricos como factores pronósticos en la presencia de depresión posparto, los trastornos psicológicos y las alteraciones en el período posparto son problemas comunes que encuentran en las mujeres. La dificultad más común es Baby blues, seguida de depresión posparto y trastornos de ansiedad (요)

El historial familiar de depresión en parientes de primer grado resultó significativa $(p=0.04)$ en relación con la depresión posparto, del $26.7 \%$ de mujeres que refirieron tener historial familiar de depresión, el 7,07\% de ellas presenta un indicador probable de depresión posparto; en contraste con los resultados del estudio de Ana María Poo et al, en los que el $50 \%$ de mujeres encuestadas tienen familiares de primer grado que han padecido cuadros depresivos, también en el estudio de O'hara M, Neunaber D, Zekoski E., hallaron que el hecho de tener antecedentes de trastornos del ánimo en familiares de primer grado aumenta las probabilidades de desarrollar depresión posparto (푸)

En cuanto a la relación entre abortos anteriores y depresión posparto se encontró que el $46 \%$ de las mujeres encuestadas tuvo entre 1 y 2 perdidas previas, de las cuales 10,6\% tuvieron una puntuación mayor a 10, dicha variable no resultó significativa $(p=0,12)$. Sin embargo, en un estudio realizado en Estados Unidos arrojó que las mujeres con pérdida previa del embarazo tenían más probabilidades de ser diagnosticadas con depresión mayor $(p=0.002)$ que las mujeres sin antecedentes de pérdida. Las mujeres con pérdidas múltiples tenían más probabilidades de ser diagnosticadas con depresión mayor $(p=0.047)$ y / o trastorno de estrés postraumático (Test exacto de Fisher $[\mathrm{FET}]=0.028$ ) que las mujeres con antecedentes de una pérdida de embarazo (요).

Dentro de las limitaciones del estudio está el hecho de que el instrumento se diligenció en algunas situaciones por vía telefónica, por lo tanto, fue necesario revalidar que las participantes hayan entendido todas y cada una de las preguntas planteadas, y en relación con el periodo de aplicación, este fue hasta los 6 meses luego del parto pudiendo así haberse presentado un sesgo de recuerdo. 


\section{CONCLUSIÓN}

Los resultados de este estudio realizado en Antioquia vienen a reafirmar una vez más que la prevalencia de la depresión posparto en Colombia es elevada, y que los problemas psicológicos, los antecedentes personales de depresión y el historial familiar de depresión en parientes de primer grado son factores que se asocian con los puntajes obtenidos por la escala. La presencia de estos factores varía entre poblaciones y en diferentes periodos de tiempo, por lo tanto, se recomienda la valoración periódica y la detección temprana en población de riesgo desde la planificación familiar o durante el control prenatal. Esto fortalecerá la planificación e intervenciones encaminadas a la prevención, tratamiento y la reducción de las posibles secuelas a la salud física y mental de la diada madre - hijo.

Contribución de los autores: G.J.H., contribuyó con la conceptualización, metodología, curación y análisis de los datos, escritura, revisión, edición; Y.P.J., contribuyó con la escritura, revisión y edición. Las estudiantes K. A. V., M.O.P., J.G.S, apoyaron en la recolección de la información y escritura. Todos los autores han leído y aceptado la versión publicada del manuscrito.

Fondos: Esta investigación no recibió fondos externos.

Conflictos de intereses: Los autores declaran no tener ningún conflicto de intereses.

\section{REFERENCIAS}

1. Silva R, Jansen K, Souza L, Quevedo L, Barbosa L, Moraes I, et al. Sociodemographic risk factors of perinatal depression: a cohort study in the public health care system. Revista Brasileira de Psiquiatria. 2012;34(2):143-8. DOI: 10.1590/s1516-44462012000200005

2. Asociación Americana de Psiquiatría (APA). Actualización del DSM-5® septiembre 2016. Man diagnósrico y estadístico Trastor Ment [Internet]. 2016;15-21. Available from: http://psychiatryonline.org/pb-assets/dsm/update/Spanish DSM5Update2016.pdf

3. Gregoire AJP, Kumar R, Everitt B, Henderson AF, Studd JWW. Transdermal oestrogen for treatment of severe postnatal depression. Lancet. 1996;347(9006):930-3. DOI: 10.1016/s0140$\underline{6736(96) 91414-2}$

4. Parsons CE, Young KS, Rochat TJ, Kringelbach ML, Stein A. Postnatal depression and its effects on child development: A review of evidence from low- and middle-income countries. Br Med Bull. 2012;101(1):57-79. DOI: $10.1093 / \mathrm{bmb} / \mathrm{ldr047}$

5. Dennis CL, Falah-Hassani K, Shiri R. Prevalence of antenatal and postnatal anxiety: Systematic review and meta-analysis. Br J Psychiatry. 2017;210(5):315-23. DOI: 10.1192/bjp.bp.116.187179

6. Gómez-Restrepo C. La Encuesta Nacional de Salud Mental-ENSM 2015. 5th ed. Vol. 45, Revista Colombiana de Psiquiatria. Santa Fe de Bogotá; 2016. 43-60 p. Disponible en: https://www.redalyc.org/pdf/806/80650839002.pdf 
7. Stephen S Lim $\ddagger$, et al. A comparative risk assessment of burden of disease and injury attributable to 67 risk factors and risk factor clusters in 21 regions, 1990-2010: a systematic analysis for the Global Burden of Disease Study 2010. Lancet. 2012;380(9859):2224-60. DOI: 10.1016/S0140$\underline{6736(12) 61766-8}$

8. Mohammad KI, Gamble J, Creedy DK. Prevalence and factors associated with the development of antenatal and postnatal depression among Jordanian women. Midwifery [Internet]. 2011;27(6):e23845. DOI: 10.1016/i.midw.2010.10.008

9. Leigh B, Milgrom J. Risk factors for antenatal depression, postnatal depression and parenting stress. BMC Psychiatry. 2008;8:1-11. DOI: 10.1186/1471-244X-8-24

10. Gelaye B, Rondon M, Araya R, Williams MA. Epidemiology of maternal depression, risk factors, and child outcomes in low-income and middle-income countries. 2016;3(10):973-82. DOI: 10.1016/S2215-0366(16)30284-X

11. Stewart DE, Robertson E, Phil M, Dennis C, Grace SL, Wallington T. Postpartum Depression: Literature review of risk factors and interventions. WHO Publ [Internet]. 2003;(October):289. Disponible en: http://www.who.int/mental health/prevention/suicide/lit review postpartum depression.pdf

12. Camacho RS, Cantinelli FS, Ribeiro CS, Cantilino A, Gonsales BK, Braguittoni É, et al. Transtornos psiquiátricos na gestação e no puerpério: Classificação, diagnóstico e tratamento. Rev Psiquiatr Clin. 2006;33(2):92-102. DOI: 10.1590/S0101-60832006000200009

13. Cox J., Holden JM, Sagovski R. Edinburgh Post-natal Depression Scale. (EPDS). Br J Psychiatry. 1987;151(10):865. Disponible en: https://www.fresno.ucsf.edu/pediatrics/downloads/edinburghscale.pdf

14. Gutierrez-Zotes A, Gallardo-Pujol D, Labad J, Martín-Santos R, García-Esteve L, Gelabert E, et al. Factor structure of the Spanish version of The Edinburgh Postnatal Depression Scale. Actas Esp Psiquiatr. 2018;46(5):174-82. https://www.actaspsiquiatria.es/repositorio/20/115/ENG/20-115-ENG174-82-749479.pdf

15. Campo A, Ayola C, Peinado H, Amor M, Cogollo Z. Escala de Edinburgh para depresion postparto: consistencia interna y estructura factorial en mujeres embarazadas de Cartagena, Colombia. Rev Colomb Obstet Ginecol. 2007;58(4):277-83. DOI: 10.18597/rcog.437

16. Rich-Edwards JW, Kleinman K, Abrams A, Harlow BL, McLaughlin TJ, Joffe H, et al. Sociodemographic predictors of antenatal and postpartum depressive symptoms among women in a medical group practice. J Epidemiol Community Health. 2006;60(3):221-7. DOl: $\underline{10.1136 / j e c h .2005 .039370}$

17. José UM, Ana RS, José GI, Mery G V., Nasser BZ, Alfi CB. Factores de riesgo de depresión posparto en puérperas venezolanas valoradas por medio de la escala de Edimburgo. Rev Chil Obstet Ginecol. 2011;76(2):102-12. DOI: 10.4067/S0717-75262011000200007

18. Khalifa DS, Glavin K, Bjertness E, Lien L. Postnatal depression among Sudanese women: Prevalence and validation of the Edinburgh Postnatal depression scale at 3 months postpartum. Int $\mathrm{J}$ Womens Health. 2015;7:677-84. DOI: 10.2147/IJWH.S81401

19. Lanes A, Kuk JL, Tamim H. Prevalence and characteristics of Postpartum Depression symptomatology among Canadian women: A cross-sectional study. BMC Public Health [Internet]. 2011;11(1):302. Disponible en: http://www.biomedcentral.com/1471-2458/11/302 
20. Giri RK, Khatri RB, Mishra SR, Khanal V, Sharma VD, Gartoula RP. Prevalence and factors associated with depressive symptoms among post-partum mothers in Nepal. BMC Res Notes. 2015;8(1):1-7. DOI: 10.1186/s13104-015-1074-3

21. Kettunen P, Koistinen E, Hintikka J. The Connections of Pregnancy-, Delivery-, and InfantRelated Risk Factors and Negative Life Events on Postpartum Depression and Their Role in First and Recurrent Depression. Depress Res Treat. 2016;2016:6-9. DOI: 10.1155/2016/2514317

22. Räisänen S, Lehto SM, Nielsen HS, Gissler M, Kramer MR, Heinonen S. Fear of childbirth predicts postpartum depression : a population-based analysis of 511422 singleton births in Finland. 2013;1-7. DOI: 10.1136/bmjopen-2013-004047

23. Liu S, Yan Y, Gao X, Xiang S, Sha T, Zeng G, et al. Risk factors for postpartum depression among Chinese women: Path model analysis. BMC Pregnancy Childbirth. 2017;17(1):1-7. https://bmcpregnancychildbirth.biomedcentral.com/articles/10.1186/s12884-017-1320-x

24. David A. Savitz CRS. The Epidemiology of Hospitalized Postpartum Depression in New York State, 1995-2004. Ann Epidemiol. 2012;21(6):399-406. DOI: 10.1016/j.annepidem.2011.03.003

25. Sierra Manzano JM, Carro García T, Ladrón Moreno E. Variables asociadas al riesgo de depresión posparto. Edinburgh Postnatal Depression Scale. Aten Primaria [Internet]. 2002;30(2):10311. Disponible en: http://dx.doi.org/10.1016/S0212-6567(02)78979-8

26. Rincón-Pabón D, Ramírez-Vélez R. Depresión posparto en mujeres colombianas: Análisis secundario de la Encuesta Nacional de Demografía y Salud-2010. Rev Salud Publica. 2014;16(4):53446. DOI: $10.15446 /$ rsap.v16n4.40673

27. Alharbi AA, Abdulghani HM. Risk factors associated with postpartum depression in the Saudi population. Neuropsychiatr Dis Treat. 2014;10:311-6. DOI: 10.2147/NDT.S57556

28. Bener A, Gerber LM, Sheikh J. Prevalence of psychiatric disorders and associated risk factors in women during their postpartum period: a major public health problem and global comparison. Int $J$ Womens Health. 2012;4:191-200. DOI: 10.2147/IJWH.S29380

29. Park JH, Karmaus W, Zhang H. Prevalence of and risk factors for depressive symptoms in Korean women throughout pregnancy and in postpartum period. Asian Nurs Res (Korean Soc Nurs Sci) [Internet]. 2015;9(3):219-25. Available from: DOl: 10.1016/j.anr.2015.03.004

30. Taherifard P, Delpisheh A, Shirali R, Afkhamzadeh A, Veisani Y. Socioeconomic, psychiatric and materiality determinants and risk of postpartum depression in border city of llam, western Iran. Depress Res Treat. 2013;2013. DOI: 10.1155/2013/653471

31. Milgrom J, Hirshler Y, Reece J, Charlene CH, Gemmill Alan AW. Social support-a protective factor for depressed perinatal women? Int J Environ Res Public Health. 2019;16(8). DOI: $\underline{10.3390 / i j e r p h 16081426}$

32. Rojas G, Fritsch R, Guajardo V, Rojas F, Barroilhet S, Jadresic E. Caracterización de madres deprimidas en el posparto. 2010;536-42. DOI: 10.4067/S0034-98872010000500002

33. Alvarado-esquivel C, Sifuentes-álvarez A, Estrada-martínez S, García-lópez CR, Sandovalherrera F. Prevalencia de depresión posnatal en mujeres atendidas en hospitales públicos de Durango, México. 2010;146(1):1-9. Disponible en: https://www.medigraphic.com/cgibin/new/resumen.cgi?IDARTICULO=24473 
34. Canaval GE, Ph D, González MC, Martínez-schallmoser L, Ph D, Tovar MC, et al. Depresión postparto, apoyo social y calidad de vida en mujeres de Cali, Colombia. Colombia Médica. 2000;31:410. Disponible en: https://www.redalyc.org/articulo.oa?id=28331102

35. Machado Ramírez F, García Serrano T, Moya Rüegg N B, Sáez N CDR. Depresión puerperal. Factores asociados. Aten. Primaria; p. 161-6. Disponible en: https://www.elsevier.es/es-revistaatencion-primaria-27-articulo-depresion-puerperal-factores-relacionados-14610

36. Navarrete RG, López LG. Depresión posparto y factores asociados en población derechohabiente del ISSSTE en Yucatán. :33-45. Disponible en: http://www.cirsociales.uady.mx/revUADY/pdf/262/ru2625.pdf

37. H Tambağ ZT, Tolun S, R Can. Perceived Social Support and Depression Levels of Women in the Postpartum Period in Hatay, Turkey. Niger J Clin Pract. 2018;21:1525-30. Disponible en: http://www.njcponline.com/article.asp?issn=1119-

3077; year=2018; volume $=21 ;$ issue $=11 ;$ spage $=1525$; epage $=1530 ;$ aulast=Tambag

38. Giannandrea SAM, Cerulli C, Anson E, Chaudron LH. Increased risk for postpartum psychiatric disorders among women with past pregnancy loss. J Women's Heal. 2013;22(9):760-8. DOI: 10.1089/jwh.2012.4011

39. Poó AM, Espejo S C, Godoy P C, Gualda de la C M, Hernández O T. Prevalencia y factores de riesgo en puérperas de un Centro de. Rev Méd Chile. 2008;135:44-52. DOI: 10.4067/S0034$\underline{98872008000100006}$ 